\title{
The world's smallest capacitive dilatometer, for high-resolution thermal expansion and magnetostriction in high magnetic fields
}

Cite as: Rev. Sci. Instrum. 88, 083903 (2017); https://doi.org/10.1063/1.4997073

Submitted: 16 May 2017 . Accepted: 19 July 2017 . Published Online: 08 August 2017

R. Küchler, A. Wörl, P. Gegenwart, M. Berben, B. Bryant, and S. Wiedmann

COLLECTIONS

EP This paper was selected as an Editor's Pick
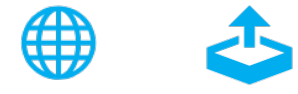

\section{ARTICLES YOU MAY BE INTERESTED IN}

A compact and miniaturized high resolution capacitance dilatometer for measuring thermal expansion and magnetostriction

Review of Scientific Instruments 83, 095102 (2012); https://doi.org/10.1063/1.4748864

A uniaxial stress capacitive dilatometer for high-resolution thermal expansion and magnetostriction under multiextreme conditions

Review of Scientific Instruments 87, 073903 (2016); https://doi.org/10.1063/1.4958957

Versatile and compact capacitive dilatometer

Review of Scientific Instruments 77, 123907 (2006); https://doi.org/10.1063/1.2403088

\begin{tabular}{|c|c|c|c|c|}
\hline & $\begin{array}{c}\text { Nanopositioning } \\
\text { Systems }\end{array}$ & $\begin{array}{c}\text { Modular } \\
\text { Motion Control }\end{array}$ \\
\hline MAD CITY LAES INC. \\
Www.madcitylabs.com
\end{tabular}




\title{
The world's smallest capacitive dilatometer, for high-resolution thermal expansion and magnetostriction in high magnetic fields
}

\author{
R. Küchler, ${ }^{1,2}$ A. Wörl, ${ }^{2}$ P. Gegenwart, ${ }^{2}$ M. Berben, ${ }^{3}$ B. Bryant, ${ }^{3}$ and S. Wiedmann ${ }^{3}$ \\ ${ }^{1}$ Max Planck Institute for Chemical Physics of Solids, Nöthnitzer St. 40, 01187 Dresden, Germany \\ ${ }^{2}$ Experimental Physics VI, Center for Electronic Correlations and Magnetism, University of Augsburg, \\ Universitätsstrasse 2, 86135 Augsburg, Germany \\ ${ }^{3}$ High Field Magnet Laboratory (HFML-EMFL) and Institute for Molecules and Materials, Radboud University, \\ Toernooiveld 7, 6525 ED Nijmegen, The Netherlands
}

(Received 16 May 2017; accepted 19 July 2017; published online 8 August 2017)

\begin{abstract}
For the characterization of novel quantum phases of matter, it is often required to study materials under multi-extreme conditions, in particular down to very low temperatures and in very high magnetic fields. We developed the world's smallest high-resolution capacitive dilatometer suitable for temperatures down to $10 \mathrm{mK}$ and usage in high magnetic fields up to $37.5 \mathrm{~T}$. Despite the extreme miniaturization, the capacitive dilatometer can resolve length changes down to $0.01 \AA$. This is an unprecedented resolution in a capacitive dilatometer of this compact size. Many cryogenic devices have limited space. Due to the extremely reduced cell size $\left(3 \mathrm{~cm}^{3}, 12 \mathrm{~g}\right)$, implementation or new applications in many of these sample space lacking devices are now possible. As an important example, the minute device can now be rotated in any standard cryostat, including dilution refrigerators or the commercial physical property measurement system. The present super compact design provides also for high resolution thermal expansion and magnetostriction measurements in a $15.2 \mathrm{~mm}$ diameter tube, enabling its use in the $32 \mathrm{~mm}$ bore, $37.5 \mathrm{~T}$ Bitter magnet at the High Field Magnet Laboratory in Nijmegen down to a temperature of $300 \mathrm{mK}$. Published by AIP Publishing. [http://dx.doi.org/10.1063/1.4997073]
\end{abstract}

\section{INTRODUCTION}

The significance of thermal expansion in the research of solid bodies cannot be underestimated. The close relationship between the two thermodynamic measurements of thermal expansion and specific heat, first discovered by Grüneisen ${ }^{1}$ in 1912, has meanwhile been developed into a comprehensive scientific theory. ${ }^{2-5}$ The characterization of phase transitions using Ehrenfest and Maxwell relations leads to a better understanding of the many interesting states exhibited by new types of materials. The linear coefficient $\alpha=L^{-1}(d L / d T)_{p}$ describes how the length of a piece of given material changes with temperature $(T)$ at constant pressure $(p)$. The volume expansion coefficient $\beta$, which for isotropic materials is given by $3 \times \alpha$, quantifies the relative change of the sample volume $V$ with temperature. Using Maxwell's relation, the volume thermal expansion coefficient can be expressed as $\beta=-V^{-1}(d S / d p)_{T}$, where $S$ and $p$ denote entropy and pressure, respectively. A study of single crystalline samples shows dilatometry to have a decisive advantage over the measurement of specific heat, $C=T(d S / d T)_{p}$ : depending on the crystal symmetry, different values sometimes result for the linear thermal expansion along the various crystal axes. This enables a more comprehensive study of the crystal structure since complementary directional dependent information is obtained. For second-order phase transitions, the Ehrenfest relation

$$
\left(d T_{c} / d p\right)_{p \rightarrow 0}=V_{m o l} T_{c} \Delta \beta / \Delta C
$$

allows us to calculate the initial pressure dependence of the phase transition temperature from the ratio of the discontinuities in thermal expansion $(\Delta \beta)$ and specific heat $(\Delta C)$. Here, also different pressure dependencies can exist for the various crystal axes. Thermal expansion is therefore a very important and sensitive tool for investigating all types of phase transitions in condensed matter.

Furthermore, thermal expansion is particularly interesting for studying the low-temperature behavior of correlated electron systems. For materials near a quantum critical point (QCP), the thermal expansion coefficient diverges much more strongly than the specific heat as $T \rightarrow 0$ and therefore its measurement can be considered as a smoking gun experiment. Scaling analysis ${ }^{6}$ revealed that the Grüneisen ratio $\Gamma=\beta / C$ has to diverge at any pressure driven QCP with $\Gamma=1 / T^{\epsilon}$, where the critical exponent $\epsilon$ allows the characterization of the nature of the QCP. ${ }^{7-9}$

While the measurement of specific heat is a standard method of investigation in most research facilities, dilatometry studies have remained relatively rare. This is primarily due to the fact that the measurement of thermal length change is more complicated and the development of such a measuring device is considerably more challenging. We described a new innovative manufacturing process in Ref. 10, which can be used to produce an extremely compact and easily operated highresolution capacitive miniature dilatometer. The dilatometers described achieve an excellent resolution of $0.02 \AA$ when a conventional commercial capacitive measuring bridge with $10^{-6} \mathrm{pF}$ resolution is used. The size of the described miniature dilatometer is (height $\times$ width $\times$ depth $=2.3 \mathrm{~cm} \times 2.6 \mathrm{~cm}$ $\times 2 \mathrm{~cm})=12 \mathrm{~cm}^{3}$. It is therefore already one of the world's smallest high-resolution dilatometers. According to statements in the literature, even smaller but still highresolution capacitive dilatometers have been developed solely by Neumeier et al. ${ }^{11}(2 \mathrm{~cm} \times 2 \mathrm{~cm} \times 1.8 \mathrm{~cm})=7.2 \mathrm{~cm}^{3}$, by Schmiedeshoff et al. ${ }^{12}(1.9 \mathrm{~cm} \times 1.9 \mathrm{~cm} \times 1.9 \mathrm{~cm})=6.9 \mathrm{~cm}^{3}$, 
and by Rotter et al. ${ }^{13}(2.1 \mathrm{~cm} \times 2 \mathrm{~cm} \times 1.8 \mathrm{~cm})=7.6 \mathrm{~cm}^{3}$. Although two of these miniature dilatometers ${ }^{11,12}$ also achieve a resolution in the sub-angström range, none of them reach our maximum resolution of $0.02 \AA .{ }^{10}$ Since samples of innovative materials are frequently just a few millimeters or even smaller in size, the absolute length change of the sample at low temperatures tends to be extremely small, making a very high resolution of the dilatometer extremely important. The resolution of $\Delta L=10^{-2} \AA$, which is required for the study of millimeter-sized samples at low temperatures, can only be achieved by capacitive dilatometry. Piezo-cantilever technology, which can be used to achieve a resolution of $\Delta L=10^{-1} \AA$, is still the second-best option. ${ }^{14}$ When round plate capacitors with a radius $r$ are used, the resolution is directly proportional to the plate radius squared $\left(\Delta L \propto r^{-2}\right)$. This correlation demonstrates the great difficulty in the development of high-resolution but also miniaturized dilatometers. Miniaturization is generally accompanied by a considerable reduction in measurement precision. Although the miniature dilatometers described above are already very small, they are still too large for certain applications due to the limited size of cryogenic devices. We have completely revamped our first dilatometer design ${ }^{10}$ and continued to optimize the manufacturing method and have thus fully utilized the possibilities of the latest production technology. This has enabled us to produce the world's smallest miniature dilatometer by far, while achieving extremely high resolution. We have been able to reduce not just the size of the dilatometer by a factor of four from $12 \mathrm{~cm}^{3}(2.3 \mathrm{~cm} \times 2.6 \mathrm{~cm} \times 2 \mathrm{~cm})$ to $3 \mathrm{~cm}^{3}$ $(1.5 \mathrm{~cm} \times 1.4 \mathrm{~cm} \times 1.47 \mathrm{~cm})$ but the weight as well from $48 \mathrm{~g}$ to $12 \mathrm{~g}$. Despite the further miniaturization and the reduction in the plate radius from $r=7 \mathrm{~mm}$ to $r=5 \mathrm{~mm}$, the new dilatometers do not exhibit any significant loss in precision. In contrast, the achievable resolution at low temperatures of about $0.01 \AA$ is even slightly better than that of its larger counterparts. Both the extreme miniaturization and the excellent resolution open up whole new fields of application for this new generation of miniature dilatometers. In Sec. II, we first describe the new design. In Sec. III, we demonstrate the extraordinary sensitivity of our mini-dilatometer by showing a low-temperature thermal expansion measurement of an $\mathrm{YbNi}_{4} \mathrm{P}_{2}$ single crystal. In Chap. IV, we will then discuss the expanded range of possible applications for the miniature dilatometers in the commercial PPMS system. While the larger counterparts can only be installed vertically in the multifunction chamber, the new mini-dilatometer can be rotated manually within the chamber. This makes it possible to measure the linear thermal expansion and the magnetostriction at every angle $0^{\circ} \leq \mu \leq 90^{\circ}$ with respect to the applied magnetic field. Thus, the impact of the magnetic field on the crystal structure can be studied comprehensively. In Sec. V, we report on the usage of a high-resolution capacitive dilatometer at the High Field Magnet Laboratory (HFML) in Nijmegen. The present super compact design provides for high resolution magnetostriction measurements in a $15.2 \mathrm{~mm}$ diameter tube, enabling its use in the $32 \mathrm{~mm}$ bore, 37.5 T Bitter magnet at HFML, down to a temperature of $300 \mathrm{mK}$. With the commissioning of the new hybrid magnet in 2018 , the field range will even be expanded even further, to $45 \mathrm{~T}$.

\section{THE NEW MINI-DILATOMETER}

The mini-dilatometer was completely revamped, based on our patented design, ${ }^{10}$ which functions in accordance with the Pott-Schefzyk principle ${ }^{15}$ of two flat parallel leaf springs. While the Pott-Schefzyk dilatometer contained many individual parts, our innovative new design consists of a body (the part accounting for the actual shape and function) produced from a single piece of $\mathrm{Be}-\mathrm{Cu}$ by means of milling and spark erosion. Solely these innovations and the patented manufacturing method made it possible for us to produce the world's smallest high-resolution dilatometer by far. The new design has enabled us to reduce the spatial dimensions (height $\times$ width $\times$ depth) from $12 \mathrm{~cm}^{310}$ to $3 \mathrm{~cm}^{3}$. The weight as well could be reduced by four times from $48 \mathrm{~g}$ to $12 \mathrm{~g}$. This is accompanied by significantly enhanced thermalisation of the dilatometer. The new design is schematically represented in Fig. 1. All of the parts (except for the electrically insulating washers) were produced from ultrapure $\mathrm{Be}-\mathrm{Cu}$ to reduce eddy currents induced by the time variation of the magnetic field. The Be component of $1.84 \%$ produces an electrical conductivity which is 30 times lower than that of the pure material. The eddy currents induced with the field sweeps are much smaller than those with cells produced from pure copper or silver. The considerable reduction in the dilatometer size and weight has a favorable impact. Less material means less surface area in which the ring currents can flow. The new dilatometer design also includes many recesses: on the one hand to completely eliminate the screw heads and to thus minimize the external dimensions. On the other hand, the recesses prevent the flow of continuous ring currents and thus also minimize the formation of eddy currents.

The new dilatometer essentially consists of four parts: (a) a lower component (shown in grey in Fig. 1), which contains the lower capacitor plate (6). (b) The body (the golden center part), which contains both the two leaf springs and the upper capacitor plate (5). This body essentially defines the shape and function of the dilatometer. (c) The cover (the thin grey part), which is screwed onto the body. This cover's new design includes a lock screw (12, orange), whose function is further described below. (d) The sample-adjusting aid (11, grey). This includes a fine-threaded adjusting screw (9) which can be used to clamp the sample (4, red). The sample can be easily inserted as follows: The sample-adjusting aid (11, grey) is unscrewed and removed, the cover as well. Now the sample can be placed very easily on the center of the body from the top. Then the cover is screwed back onto the body, followed by the sampleadjusting aid. The sample is initially still freestanding and is clamped in the next step by a tightening of the adjusting screw (9). In this process, the screw does not press directly against the sample, but rather against a cubic piston (10) that can move horizontally inside the cover. This prevents the sample from twisting when it is inserted. Once the sample is clamped, the lock screw (12) is used to lock the cubic piston (10) in place. The sample-adjusting aid can then be unscrewed and removed. This innovation enables the dilatometer to achieve the desired low construction height of just $1.47 \mathrm{~cm}$. The dilatometer operates in accordance with the Pott and Schefzyk principle: ${ }^{15}$ it consists of an external C-shaped frame (3) and a mobile part 

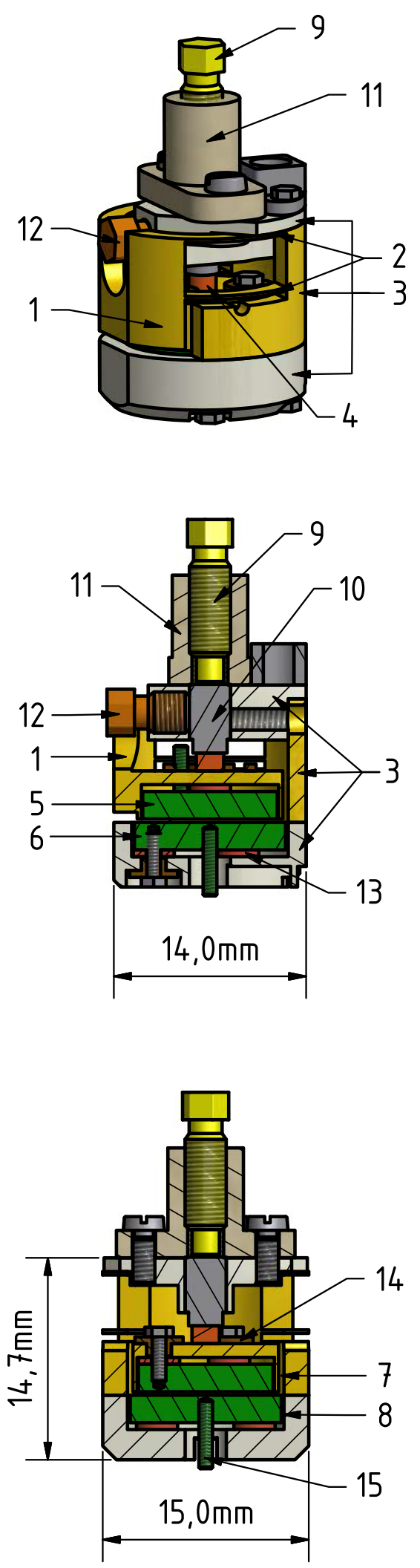

FIG. 1. A schematic view of the mini-dilatometer. The upper panel shows a 3D-view, the middle panel shows a side cut-away view, and the lower panel shows a front cut-away view of the cell. (1) mobile part, (2) Be-Cu flat springs, (3) external frame, (4) sample, (5) upper capacitor plate, (6) lower capacitor plate, (7 and 8) guard rings, (9) adjusting screw, (10) cubic piston, (11) sampleadjusting-aid, (12) lock screw, (13) sapphire-washer, (14) insulating piece of Vespel, (15) electrical connection.
(1). The lower capacitor plate (6) is located on the lower grey part of the external frame (3). The upper capacitor plate (5) was mounted at the bottom on the mobile part (1). The external frame and the mobile part are connected by two leaf springs (2) with a thickness of $0.3 \mathrm{~mm}$. The sample can be clamped between the mobile part (1) and the external frame (3) by the adjusting screw. A small force of $4 \mathrm{~N}$ against the spring force is required for this. If the length of the sample then changes during the experiment due to a temperature change or a magnetic field sweep, the distance between the capacitor plates also changes in the same manner. A commercial capacitance measuring bridge can be used to record the measured change in capacitance; the simple formula of the plate capacitor can then be applied to calculate this change in capacitance back to the length change caused by the sample. Thanks to the parallelogram suspension, the movement of the upper capacitor plate is exactly perpendicular to that of the lower plate. The new mini-dilatometer can be used to measure samples less than $1 \mathrm{~mm}$ up to $3 \mathrm{~mm}$ in size. Each of the two capacitor plates is electrically insulated by three pieces of Vespel (14) and three sapphire washers (13) and surrounded by protective rings (7 and 8) for preventing stray fields. An essential production step is the polishing of the capacitor plates installed in the protective rings. This is done after the capacitor plates have been assembled. The quality of the dilatometer greatly depends on how parallel and vertically the capacitor plates and the external contact surfaces of the housing can be polished. We have developed a patented polishing device for this purpose. Thanks to this device, we achieve a nearly absolute parallel orientation of the capacitor plates towards each other. The two capacitor plates are $0.25 \mathrm{~mm}$ apart in the initial position. This corresponds to a capacitance of around $3 \mathrm{pF}$. Once the sample has been inserted, an adjusting screw (9) is used to clamp the sample even tighter. All of the mini-dilatometers manufactured up to now cause a short circuit at a capacitance exceeding 30-50 pF. This makes it possible to work with a very high measuring capacitance between 10 and $20 \mathrm{pF}$. At a measuring capacitance of $20 \mathrm{pF}$, the two capacitor plates are merely $0.025 \mathrm{~mm}$ apart and a force of $4 \mathrm{~N}$, which is negligible for most materials, is applied to the sample. At such a high measuring capacitance, the absolute value of the capacitance can be measured with a resolution of $10^{-6} \mathrm{pF}$, which corresponds to a sensitivity of $\Delta L=0.01 \AA$ in the absolute length change. The new mini-dilatometers are extremely high-resolution dilatometers despite their tiny dimensions.

\section{PERFORMANCE TEST IN A DILUTION REFRIGERATOR}

In this chapter, we demonstrate the extraordinary sensitivity of our mini-dilatometer by the low-temperature thermal expansion of an $\mathrm{YbNi}_{4} \mathrm{P}_{2}$ single crystal measured perpendicular to the $c$-axis with a length of $l=1.8 \mathrm{~mm}$.

To perform the experiment, a temperature regulation plate (TRP) was mounted on the mixing chamber (MC) of a dilution refrigerator. The TRP consists of a copper plate which is thermally insulated against the MC. Only a thin copper wire thermally couples the MC and TRP. The dilatometer was attached to the TRP by a slotted copper rod and cold finger (see 


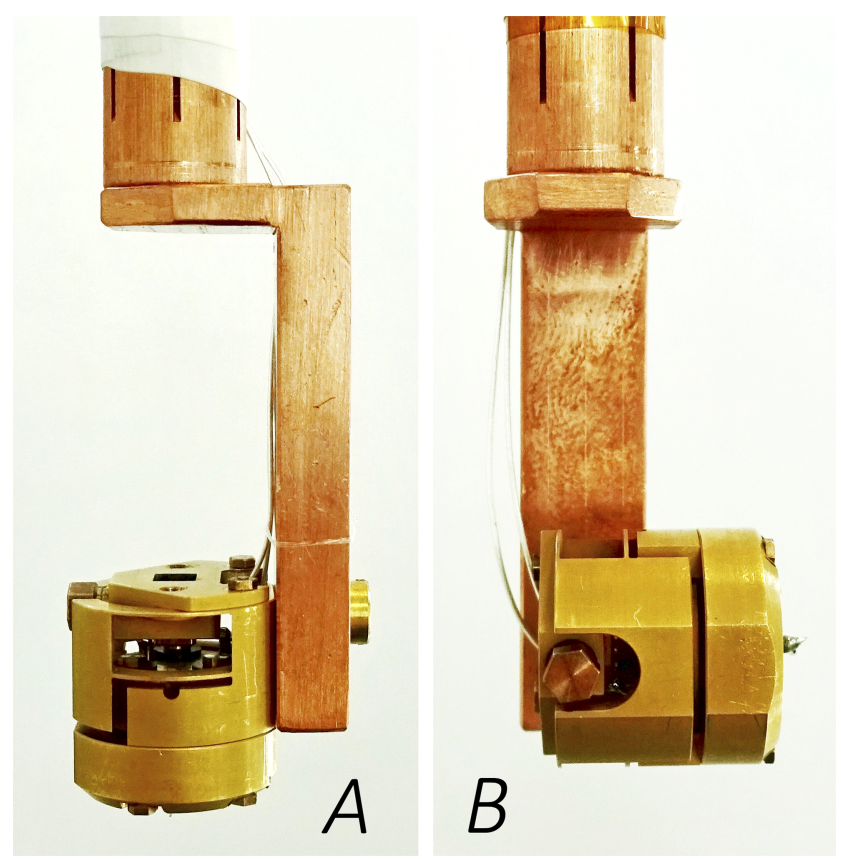

FIG. 2. Photograph of the cell mounted on a cold finger of a dilution refrigerator (a) parallel and (b) perpendicular to the applied magnetic field.

Fig. 2) to reduce eddy currents and ensure sufficient thermalization of the sample. A temperature sensor and joule heater were placed on the TRP and controlled by an LS372 resistance bridge. For the variation of temperature, an exponential temperature $\operatorname{ramp} T=T_{0} \exp (a t)$, where $T_{0}$ is the initial temperature and $a$ is the sweep rate, was used. Typical sweep rates are $|a| \leq 0.00025 \mathrm{l} / \mathrm{s}$.

At temperatures below $T_{C}=0.15 \mathrm{~K}, \mathrm{YbNi}_{4} \mathrm{P}_{2}$ is a ferromagnet. ${ }^{16}$ The sharp kink-like anomaly observed in $\Delta L / L$ at $T_{C}$ [see Fig. 3(b)] indicates the second-order phase transition. The inset shows the extraordinary sensitivity of our mini-dilatometer with a very high resolution of slightly better than $0.01 \AA$. Thermal expansion under high field (up to $10 \mathrm{~T}$ ) and magnetostriction data with the same sensitivity has been

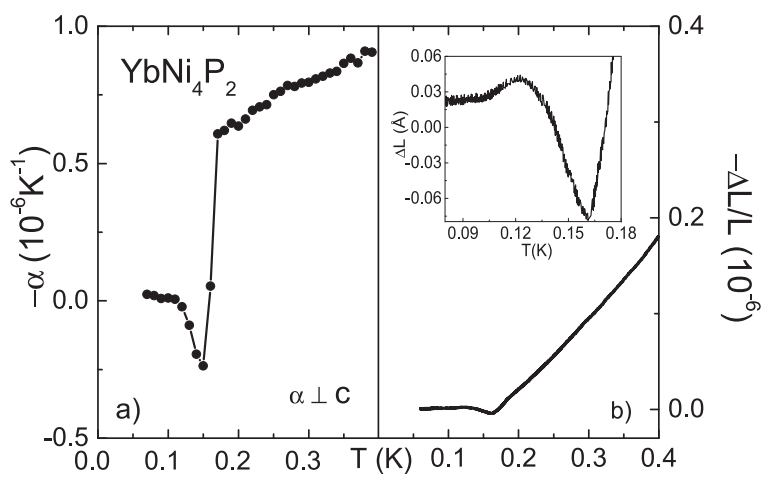

FIG. 3. Low-temperature thermal expansion coefficient $\alpha(T)$ (a) and relative length change $\Delta L / L_{0}(T)$ (b) of an $\mathrm{YbNi}_{4} \mathrm{P}_{2}$ single crystal, measured perpendicular to the $c$-axis. The sharp kink-like anomaly observed in $\Delta L / L$ at $T_{C}$ of the second order phase transition demonstrates the high sensitivity of our dilatometer and the very good quality of the sample. The inset shows the high resolution of $0.01 \AA$ at low temperatures. obtained as well. Since the noise level is extremely small, we could use a very short temperature window of $10 \mathrm{mK}$ derivative calculation to obtain the thermal expansion coefficient $\alpha(T)$ [see Fig. 3(a)]. The absolute values of $\alpha(T)$ are in agreement with a former measurement on a different single crysta ${ }^{16}$ but the feature of the observed anomaly is now more pronounced. The measurement demonstrates that, due to the extreme tiny cell size, thermal equilibrium for both the cell and the sample is reached very quickly and a complicated setup, where the sample is thermally isolated from the cell, is not necessary. The absence of hysteresis between warming and cooling confirms the rapid thermalization of the sample as well. Measurements at a high magnetic field of $10 \mathrm{~T}$ and temperature of $1 \mathrm{~K}$ show a comparable resolution of $0.02 \AA$.

\section{NEW POSSIBILITIES: (1) BROADER APPLICATION FOR PPMS USE}

In this section, we discuss the expanded range of possible applications for the new mini-dilatometers in the commercial PPMS (Physical Property Measurement System) system. All of the capacitive miniature dilatometers previously developed worldwide ${ }^{10-13}$ are of a size which could just barely fit into a PPMS application in the ideal case. The new extremely miniaturized dilatometer opens up a new possibility: it can be manually adjusted to the applied magnetic field at any desired angle between $0^{\circ} \leq \mu \leq 90^{\circ}$. Figure 4 provides an example of this for the angles $0^{\circ}, 45^{\circ}$, and $90^{\circ}$. At the moment, the angle of the mini-dilatometer can only be changed manually; however, an implementation of a Swedish rotator to the PPMS-probe is under development. This should enable us soon to rotate the dilatometer in situ.

Figure 5 shows the PPMS mini-dilatometer probe. For operation between 2 and $300 \mathrm{~K}$, the sample space was kept under a helium atmosphere of typically $1 \mathrm{mbar}$, which ensured a good thermal contact of the cell and the included sample. Two coaxial cables connect the capacitor plates with the commercial measuring bridge. The PPMS insert is thermally coupled to the annulus via a pin connector, where a heater warms the helium gas to the correct temperature. Gold plated thermal anchors mounted to a copper block (1) just above the cell connect the probe to the walls of the Inner Vacuum Chamber (IVC) improving the thermal coupling to the cell. Additional anchors (2) are mounted above the insert at several points of the probe to reduce the temperature successively from the top of the cryostat down to the cell. The heat leak caused by the coaxial cables is reduced by wrapping them around the probe.

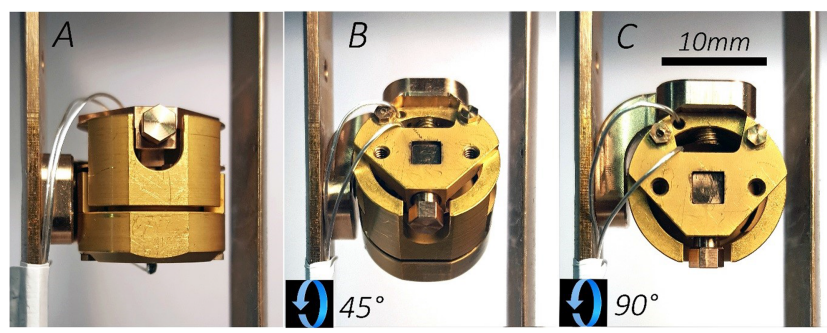

FIG. 4. Manual Rotation of a mini-dilatometer within a PPMS-probe. 

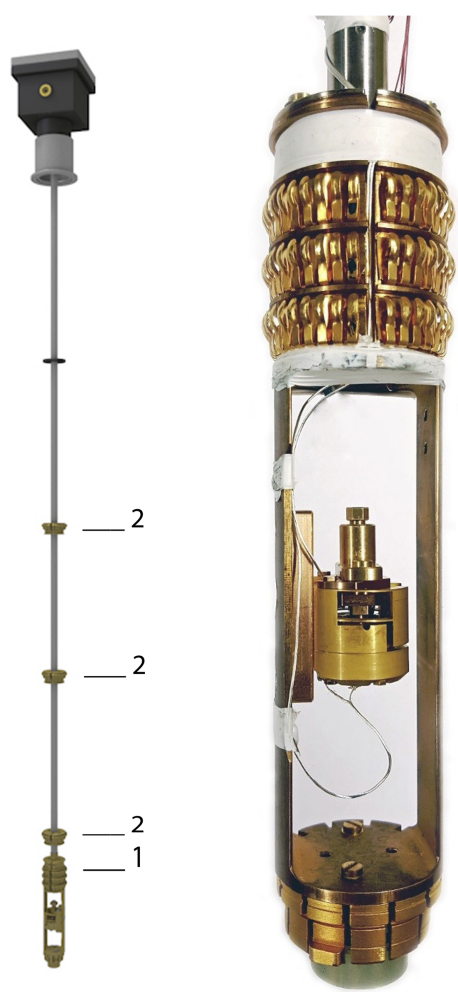

FIG. 5. Left: Drawing of the PPMS probe with gold plated thermal anchors (1 and 2) and connectors for capacitance and temperature measurements. Right: Photograph of the mini-dilatometer within the PPMS probe.

\section{A. Cell background}

As in the case of the larger dilatometer, ${ }^{10}$ the minidilatometer also has a temperature-dependent background which is formed by the thermal expansion of the various materials in the dilatometer structure. Since the mini-dilatometer was manufactured solely from $\mathrm{Cu}-\mathrm{Be}$ with the exception of the sapphire washers and the electrically insulating Vespel parts, the temperature-dependent background is small. The dilatometer's assembly bracket plays no part in this. Figure 6 shows a comparative measurement for a vertical and a horizontal dilatometer assembly. In both cases, the temperaturedependent background is extremely small up to $30 \mathrm{~K}$, and then increases slightly and progresses nearly linearly from 50 to $300 \mathrm{~K}$. It does not exhibit any anomalies and can thus be easily calibrated. The temperature-dependent background of the mini-dilatometer hardly differs at all from that of the larger dilatometers, neither in the progression nor in the absolute values (is somewhat smaller). For this reason, the calibration and data analysis are performed in the same manner. We refer to Refs. 10,15, and 17 here, where these processes are described in detail.

The background, which depends on the magnetic field, shows a behavior which is comparable to the larger dilatometer (Sec. VI of Ref. 10). The background $\Delta L(B)$ of the empty minidilatometer shows power law behaviour with an exponent close to two: $\Delta L(B)=-3 \times 10^{-9} \mathrm{~cm} \times B(T)^{1.7}$.

\section{B. PPMS performance test on a $\mathrm{CoS}_{2}$ single crystal}

The sample temperature was measured by use of a calibrated Cernox sensor, which was fixed on top of the

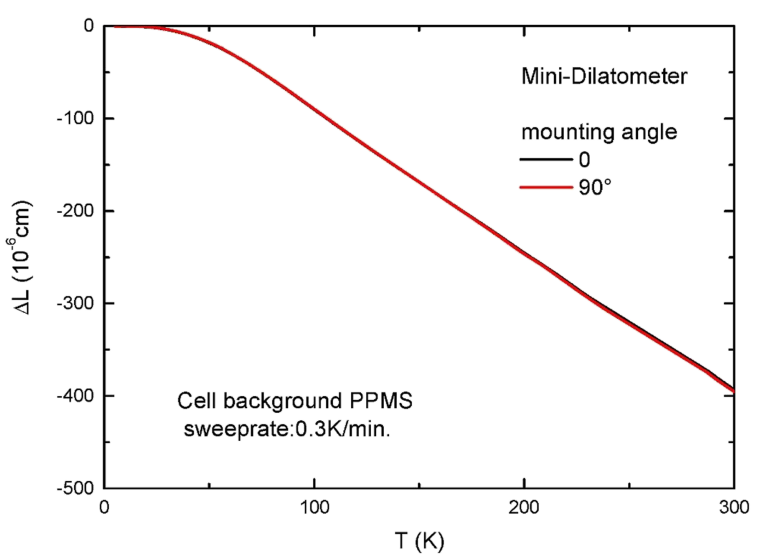

FIG. 6. Cell-background of a comparative measurement for a $0^{\circ}$ and $90^{\circ}$ dilatometer assembly.

dilatometer. Besides, the PPMS provides a second thermometer close to the pin connector at the bottom of the system. When we increased the temperature from $2 \mathrm{~K}$ up to $300 \mathrm{~K}$ linear with $0.3 \mathrm{~K} / \mathrm{min}$., we observed a small maximum temperature difference of $0.5 \mathrm{~K}$ between the two sensors at the highest temperature. Figure 7 demonstrates the measurement quality of the mini-dilatometer in the PPMS by showing the relative length change and the determined thermal expansion coefficient of a $1 \mathrm{~mm}$ large $\mathrm{CoS}_{2}$ single crystal between 110 and $140 \mathrm{~K}$ under zero and various magnetic fields. At zero field and temperatures below $T_{C} \approx 122 \mathrm{~K}, \mathrm{CoS}_{2}$ is a half-metallic ferromagnet with a cubic pyrite structure. ${ }^{18}$ During heating, a sharp step-like anomaly, observed in $\Delta L / L_{0}(T)$ at $T_{C}=122 \mathrm{~K}$, indicates the first-order character of the phase transition. This corresponds to the sharp $\lambda$-type anomaly in $\alpha(T)$ at $T_{C}$. The absolute values as well as the observed features are in good agreement with former results by Ref. 18, which measured a very large $(8 \mathrm{~mm})$ polycrystalline sample. The small width of the anomaly in $\alpha(T)$ proofs the very good quality of the sample. Besides, it shows that the minute size of the dilatometer $\left(3 \mathrm{~cm}^{3}, 12 \mathrm{~g}\right.$ ) enables the cell, and thus also the sample, to

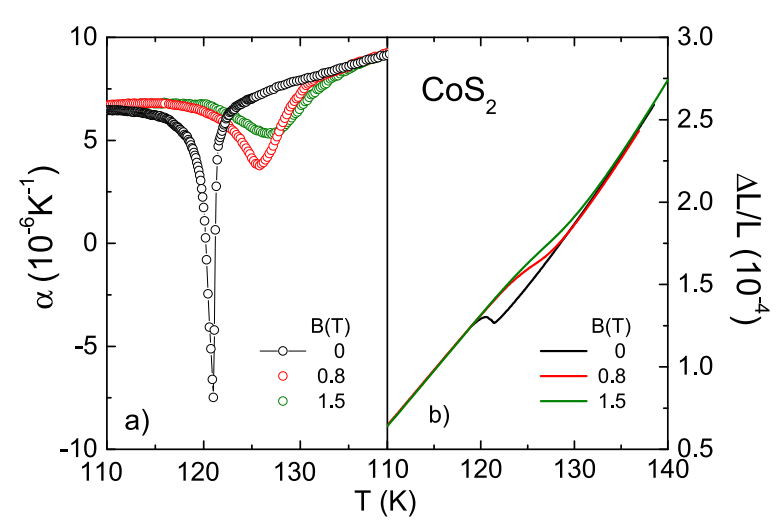

FIG. 7. (a) Thermal expansion coefficient $\alpha(T)$ and (b) relative thermal length change $\Delta L / L_{0}(T)$ of a $1 \mathrm{~mm}$ large $\mathrm{CoS}_{2}$ single crystal under magnetic field. In zero field, a clear step-like feature in $\Delta L / L_{0}(T)$ (b) and a corresponding sharp $\lambda$-type anomaly in $\alpha(T)$ at $T_{C}$ (a) can be observed. This proves the existence of a first order phase transition and demonstrates the high sensitivity of our dilatometer and the very good quality of the sample. Under magnetic field, the transition anomaly in $\alpha(T)$ broadens and $T_{C}$ shifts to higher temperature. 
quickly reach thermodynamic equilibrium. Within the PPMS, we reach an absolute resolution in $\Delta L$ of $0.1 \AA$ below $150 \mathrm{~K}$. Close to room temperature, the resolution increases slightly. The temperature dependence of the linear thermal expansion coefficient $\alpha(T)$ at various magnetic fields is also shown in Fig. 7, in which the direction of the measurement of length is parallel to the magnetic field. As can be seen, with increasing magnetic field, the sharp phase transition anomaly becomes more and more smeared out and shifts to higher temperature. This magnetic field effect on the temperature dependence and on the absolute values of $\alpha(T)$ did not change, when the thermal expansion was measured in a direction perpendicular to the magnetic field. We could also resolve magnetostriction data up to $10 \mathrm{~T}$ at $2 \mathrm{~K}$ with a absolute resolution in $\Delta L$ in the range $0.1-0.3 \AA$.

\section{NEW POSSIBILITIES: (2) HIGH RESOLUTION MAGNETOSTRICTION AND THERMAL EXPANSION IN HIGH MAGNETIC FIELDS}

Capacitive dilatometry is the standard method for measuring thermal expansion and magnetostriction in DC (continuous field) magnets. The main advantages are the high measurement resolution and the low uniaxial pressure applied to the sample. This technology has also been employed in pulsed magnetic fields but with a resolution of $\Delta L / L$ of just $10^{-5}$ which is not high enough for many applications. ${ }^{19}$ Here the method using fiber Bragg gratings is more often preferred for the measurement of magnetostriction. ${ }^{20}$ To date, capacitive dilatometers have found only limited application in water-cooled resistive magnets with fields of $30 \mathrm{~T}$ and above (Refs. 21 and 22), for various reasons. This type of dilatometer is sensitive to the mechanical and the electrical noise generated by the resistive or hybrid magnet. The greatest obstacle, however, is the very restricted space available inside the magnets which limits the size of the capacitor plates used in the dilatometer. The new mini-dilatometer is ideally suited to limited spaces, and it is also compact and sturdy and thus stable against mechanical vibrations. In the following, we report on the first successful implementation of our mini-dilatometer in a static high-field laboratory. Since we initially tested the larger dilatometer in a high field up to $30 \mathrm{~T}$ in 2015 , we will also discuss that in this context.

\section{A. Setup for experiments in high fields}

In this section, we describe the new setup for dilatometry in high magnetic fields. We have successfully implemented the mini-dilatometer and its larger counterpart at the High Field Magnet Laboratory (HFML) in Nijmegen. First the characteristics of the resistive Bitter magnets, cryostats, and vacuum tube that are available at the HFML are summarized. Then the design of new probes for both dilatometers is described. Finally the performance of the dilatometers at high magnetic field is characterized, and the scope for future developments is noted.

\section{Bitter magnet and cryogenics}

All the water-cooled, resistive Bitter magnets at the HFML have a similar design. The magnets have room temperature bore sizes of either $50 \mathrm{~mm}$ or $32 \mathrm{~mm}$. The $50 \mathrm{~mm}$ bore magnet (cell 1) can reach a static field of $30 \mathrm{~T}$; there are two $32 \mathrm{~mm}$ bore, $33 \mathrm{~T}$ magnets and a $32 \mathrm{~mm}$ bore, $37.5 \mathrm{~T}$ magnet [cell 2: (Refs. 23 and 24)]. The larger dilatometer ${ }^{10}$ can only be used in the $50 \mathrm{~mm}$ bore magnet; the new mini-dilatometer can fit inside a vacuum tube used in the $32 \mathrm{~mm}$ bore magnets ( 33 and $37.5 \mathrm{~T})$.

To minimize vibrational noise in experiments, the cryostat is mounted on a platform surrounding the magnet, independently fixed to the floor and not touching the magnet itself. Active vibration dampers decouple the cryostat from the platform: active damping functions up to $200 \mathrm{~Hz}$, and above this frequency damping is passive. The cryostat itself consists of concentric liquid nitrogen and liquid $4 \mathrm{He}$ baths. By pumping the $4 \mathrm{He}$ bath, the system can reach $1.3 \mathrm{~K}$. The dilatometer mounted on the probe is inserted into a vacuum tube that is inserted into the cryostat. The vacuum tube is double-walled, providing an inner vacuum chamber (IVC) that decouples the probe from the $4 \mathrm{He}$ bath, enabling us to either perform experiments down to $3 \mathrm{He}$ temperature $(0.3 \mathrm{~K})$ or up to room temperature (via a strain gauge heater controlled by a Lakeshore temperature controller). For the $32 \mathrm{~mm}$ bore magnets (e.g., the $37.5 \mathrm{~T}$ cell 2 ), the inner diameter of the vacuum tube is just $15.2 \mathrm{~mm}$.

\section{Probes}

Both probes are equipped with semi-rigid coaxial cables to provide good dielectric properties and shielding quality. The dilatometer at the bottom of the probe is connected with flexible coax cables to the semi-rigid one using commercially available SMX connectors. A thermometer (Cernox-CX1030 series) and a heater ( $120 \Omega$ strain gauge) are placed in the vicinity of the sample on the dilatometer body to make sure that it is in thermal equilibrium with the dilatometer.

\section{Larger dilatometer used in cell $1(50 \mathrm{~mm}, 30 \mathrm{~T})$}

The larger dilatometer has an outside diameter of $26.0 \mathrm{~mm}$. It can only be used in the $50 \mathrm{~mm}$ bore of cell 1 in a vacuum tube with an inner diameter of $28 \mathrm{~mm}$. This leaves a gap of $1 \mathrm{~mm}$. The spacer ring [(1) in Fig. 8] keeps the dilatometer from touching the tube. Touching causes extra noise in the system from vibrations in the tail of the jacket and can even cause electrical ground loops.

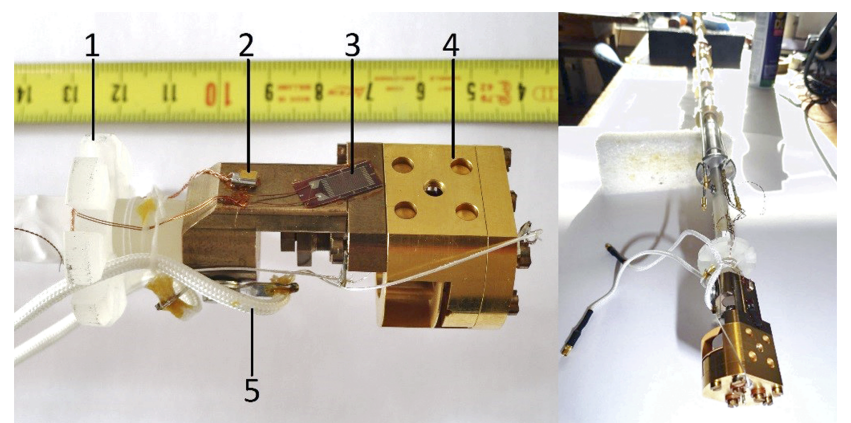

FIG. 8. Photograph of the larger dilatometer used in cell $1(50 \mathrm{~mm}, 30 \mathrm{~T})$. Left panel: Dilatometer mounted on the probe. (1) Spacer ring, (2) thermometer, (3) $120 \Omega$ strain gauge heater, (4) dilatometer body, and (5) flexible coax cable. The photographed ruler measures $\mathrm{cm}$. Right panel: View of the probe. 


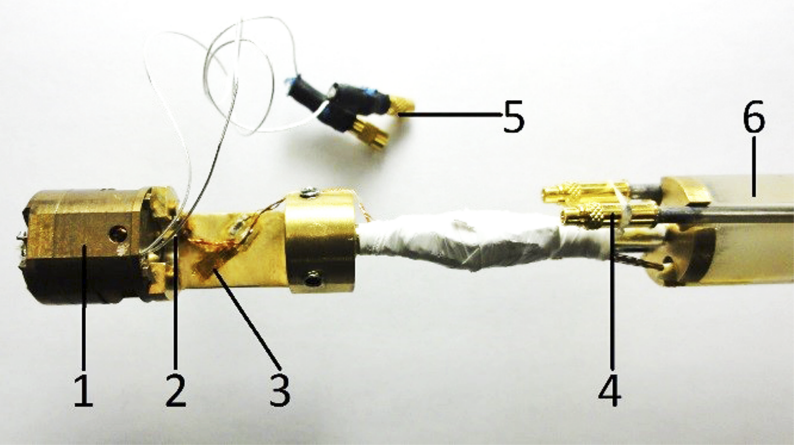

FIG. 9. Photograph of the mini-dilatometer used in cell $2(32 \mathrm{~mm}, 37.5 \mathrm{~T})$. Mini-dilatometer mounted on the probe: (1) Dilatometer body, (2) thermometer, (3) heater, (4) semi rigid coax, (5) SMX coax connector, and (6) adapter.

\section{Mini-dilatometer used in cell $2(32 \mathrm{~mm}, 37.5 \mathrm{~T})$}

Since the small dilatometer has an outside diameter of $15 \mathrm{~mm}$ and the vacuum tube has an inside diameter of $15.2 \mathrm{~mm}$, the gap is only $0.1 \mathrm{~mm}$. This was the most critical issue concerning the adapter (see Fig. 9). The main part of the adapter has been fabricated out of plastic to thermally and electrically insulate the dilatometer from the probe. The bottom part of the adapter is machined out of copper, which enables the thermal coupling to the thermometer and the heater. The adapter just fits inside the vacuum tube and ensures that the dilatometer does not touch the tube.

\section{Preliminary measurements}

In our preliminary measurements, we aim at quantifying the accessible resolution for both dilatometers up to the maximum field for both the 50 and $32 \mathrm{~mm}$ bore magnets. We derive the resolution in terms of noise $=\Delta L / L-\left(\Delta L_{\text {avg }} / L\right)$ in which avg is given by $10-50$ data points smoothed by adjacent averaging $(\approx 1 \%)$ of the total data points. While averaging, care is taken that noise does not include features such as quantum oscillations of the measured sample itself. The resolution is then derived from the width of the noise.

\section{Measurements using the larger dilatometer in cell $1(50 \mathrm{~mm}, 30 \mathrm{~T})$}

The first measurements have been carried out on a graphite (HOPG) sample with a thickness of $L_{3}=1.2 \mathrm{~mm}$ along its $c$-axis.
We use a water flow rate of $140 \mathrm{l} / \mathrm{s}$ to reach a maximum field of $30 \mathrm{~T}$. The measurements at different temperatures are displayed in Fig. 10(a). We observe an oscillatory magnetostriction in $\Delta L_{3} / L_{3}$, as expected for a diamagnetic material. ${ }^{25}$ Above $7.5 \mathrm{~T}$, all the carriers are confined to the lowest spin-split $n=0$ Landau level. Above $8 \mathrm{~T}$, we find a continuous background that follows a $-B^{1.7}$-dependence with increasing magnetic field. ${ }^{10}$ In Fig. 10(b), we present the noise level as a function of B. At zero field, we find a noise level of $\Delta L_{3} / L_{3}=3 \times 10^{-8}$ that increases gradually with increasing magnetic field. At $10 \mathrm{~T}$, we reach a noise level of around $1 \times 10^{-7}$ which remains roughly constant up to $28 \mathrm{~T}$. Most importantly, the noise level is neither significantly affected by pumping on the $4 \mathrm{He}$ bath $(1.3 \mathrm{~K})$ nor by pumping the $4 \mathrm{He}$ bath and the $3 \mathrm{He}$ separately in the vacuum tube (0.3 K).

\section{Measurements using the mini-dilatometer in cell $2(32 \mathrm{~mm}, 37.5 \mathrm{~T})$}

The mini-dilatometer was tested in cell 2, a Bitter magnet with a maximum field of 37.5 T. Measurements in cell 2 benefit from a new, more vibrationally stable mounting platform for the cryostat: this platform has been developed for high-field scanning probe microscopy measurements. Figure 11 shows the magnetostriction $\Delta L_{3} / L_{3}$ and magnetostriction coefficient $\lambda=1 / L(d \Delta L / d B)$ measured on a $L_{3}=1.7 \mathrm{~mm}$ thick graphite HOPG sample at $4.2 \mathrm{~K}$ for fields up to $37.5 \mathrm{~T}$. The water flow is $165 \mathrm{l} / \mathrm{s}$. At zero field, the noise level is $\Delta L_{3} / L_{3}=3 \times 10^{-8}$. The curve shows qualitatively the same features in magnetostriction as observed with the large dilatometer up to the quantum limit (see the inset). As can be seen in the $\lambda$ and in particular in the noise level illustrated in Fig. 12(b), the noise increases with field, particularly above $20 \mathrm{~T}$, up to $8 \times 10^{-8}$ between 20 and $30 \mathrm{~T}$ and further increases up to $1 \times 10^{-7}$ beyond $35 \mathrm{~T}$. In order to attribute the origin of the noise, we measured the vibration level using an accelerometer that was placed on top of the cryostat: vibration measurements were made at various constant magnetic fields, and the results are shown in Fig. 12(a). As can be seen, the vibration level starts to increase above $20 \mathrm{~T}$ particularly in the range between 0 and $200 \mathrm{~Hz}$. In Fig. 12(d), we show the root mean square (RMS) of the vibrational data which is cut off at a frequency of either $200 \mathrm{~Hz}$ or $1 \mathrm{kHz}$. This may be compared to the RMS of the dilatometer noise, shown in Fig. 12(c). There is a close correlation between the dilatometer noise and the RMS vibrational
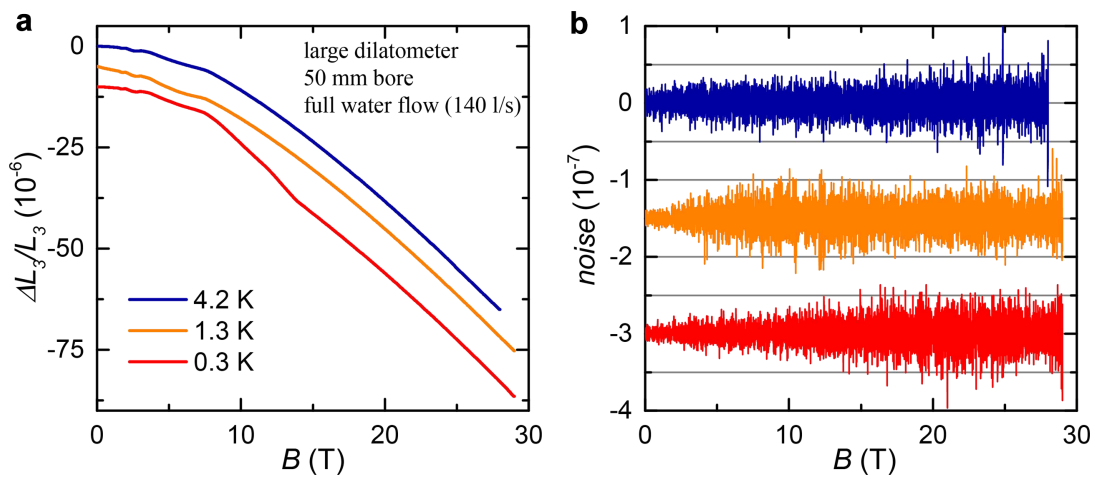

FIG. 10. (a) Magnetostriction measurements of a graphite (HOPG) sample at $0.3,1.3$, and $4.2 \mathrm{~K}$ using the large dilatometer. (b) The noise level during the measurements. 


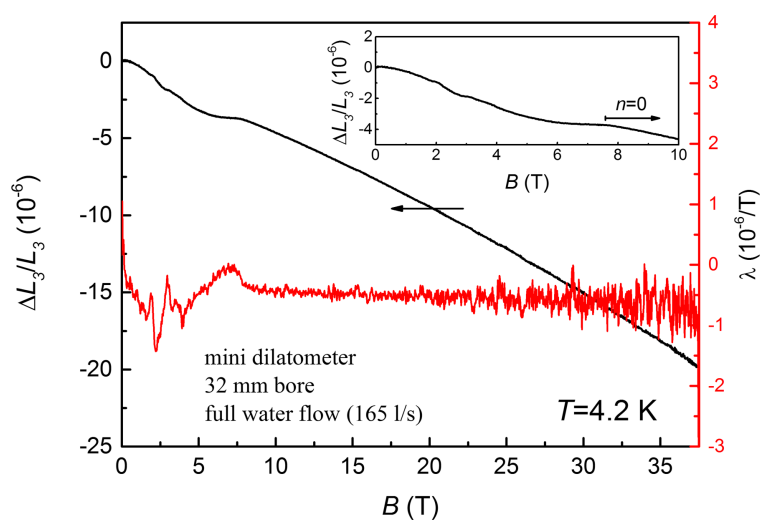

FIG. 11. Magnetostriction $\Delta L_{3} / L_{3}$ and magnetostriction coefficient $\lambda$ of a graphite sample (HOPG) at $4.2 \mathrm{~K}$ up to $37.5 \mathrm{~T}$ using the mini dilatometer. Inset: Zoom into the low-field oscillatory magnetostriction.

noise up to $200 \mathrm{~Hz}$, indicating first that the excess noise seen in the dilatometer signal at high field is likely caused by vibrational noise and second that the dilatometer is most sensitive to low-frequency vibrational noise.

Next, we compare the noise level of both dilatometers. At zero field, the noise level of the mini-dilatometer in cell 2 is
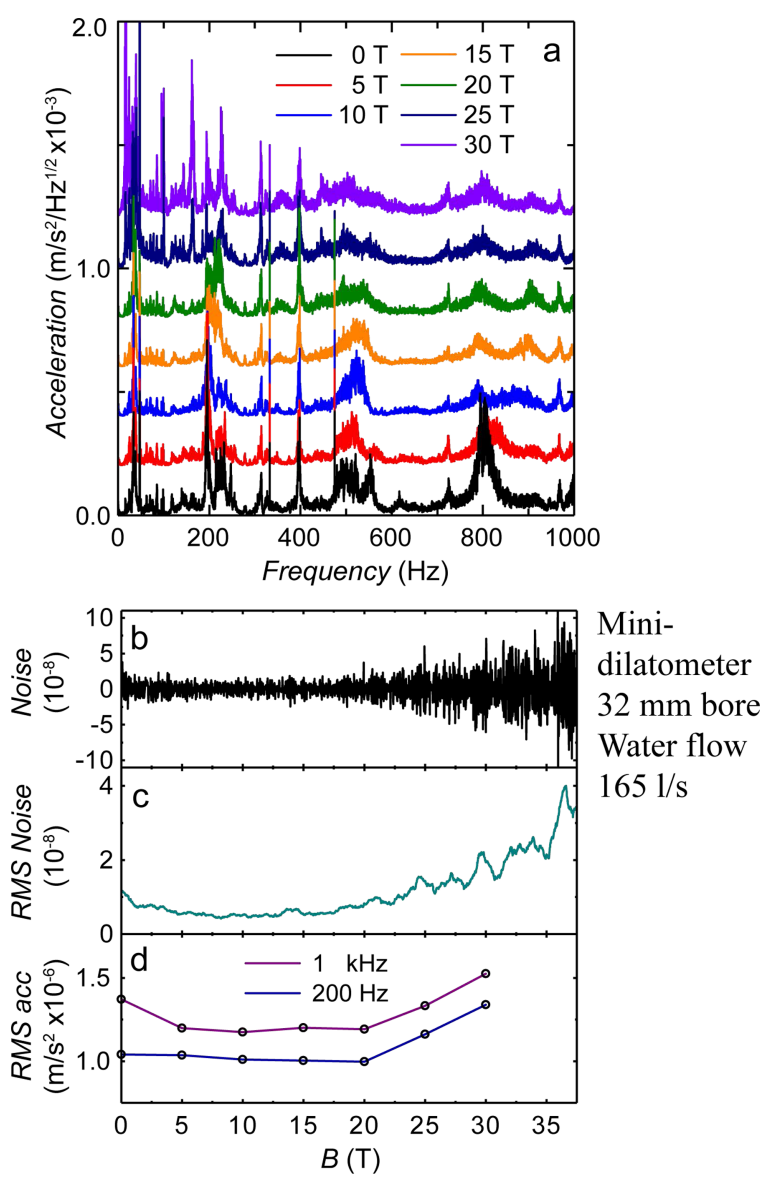

FIG. 12. Accelerometer and dilatometer noise measured in cell 2 using the mini-dilatometer up to $37.5 \mathrm{~T}$. (a) Vibrational spectra (PSD) measured at different magnetic fields using an accelerometer on top of the cryostat (curves are offset for clarity). (b) The noise level from dilatometer measurements on HOPG at $4.2 \mathrm{~K}$, from data depicted in Fig. 11. (c) Adjacent average of the RMS of the noise shown in (b). (d) RMS of the vibrational data, cut at either $200 \mathrm{HZ}$ or $1 \mathrm{kHz}$ comparable to the larger size dilatometer in cell 1 . From the noise level, we approximate a resolution of about $1 \AA$ up to $30 \mathrm{~T}$ for cell 1 . In cell 2 , above $35 \mathrm{~T}$, the noise strongly increases and we estimate a resolution $\leq 2 \AA$. The increase in noise seen in the vibration and dilatometer data in cell 2 above $20 \mathrm{~T}$ [Figs. 11 and 12(b)] can be attributed to the partly magnetic cryostat and to the action of the active vibration dampers. The cryostat tail is made of stainless steel, not fully nonmagnetic: furthermore, eddy currents in the cryostat tail are effective at coupling in vibrational noise. Thus there is an excess of noise at higher fields, which the active dampers are unable to fully compensate for leading to excess noise below $200 \mathrm{~Hz}$. Currently a nonmagnetic cryostat is in development to address these issues. We are optimistic that with the developments in hand, we will be able to perform magnetostriction measurements in the whole field range up to $37.5 \mathrm{~T}$ with an unchanged low noise level of $\leq 0.5 \AA$. Furthermore, we are currently also building a dilatometer holder for the mini-dilatometer, which will enable us to measure thermal expansion and magnetostriction perpendicular to the applied field.

\section{CONCLUSION}

We have completely revamped our first dilatometer design. Additionally, we optimized the manufacturing method and have thus fully utilized the possibilities of the latest production technology. This has enabled us to develop the world's smallest miniature dilatometer by far, while achieving extremely high resolution. We have been able to reduce not just the size of the innovative dilatometer by a factor of four from $12 \mathrm{~cm}^{3}$ to $3 \mathrm{~cm}^{3}$ but the weight as well from $48 \mathrm{~g}$ to $12 \mathrm{~g}$. Despite the further miniaturization, the new dilatometers do not exhibit any significant loss in precision. In contrast, the achievable resolution of the length changes at low temperatures of $0.01 \AA$ is even better than that of its larger counterparts. Both the extreme miniaturization and the excellent resolution open up whole new fields of application: the new device can now be rotated easily in any standard cryostat, including dilution refrigerators or the commercial physical property measurement system. The present super compact design provides also for high resolution thermal expansion and magnetostriction measurements in a $15.2 \mathrm{~mm}$ diameter tube, enabling its use in the $32 \mathrm{~mm}$ bore, $37.5 \mathrm{~T}$ Bitter magnet at the High Field Magnet Laboratory (HFML) in Nijmegen down to a temperature of $300 \mathrm{mK}$. With the commissioning of the new hybrid magnet in 2018, the field range can even be expanded to $45 \mathrm{~T}$.

\section{ACKNOWLEDGMENTS}

The authors acknowledge the assistance of Jan Gerritsen with the vibrational measurements at the HFML. We also thank T. Orton and S. Pezzini for helping with the experiments at the HFML, B. Fauque and K. Behnia for instigating the graphite project, and C. Krellner for providing the $\mathrm{YbNi}_{4} \mathrm{P}_{2}$ single crystal. We also thank M. Schmidt, who has grown the $\mathrm{CoS}_{2}$ single crystal. Continued support by A. P. Mackenzie and M. Brando is acknowledged. We are also indebted to T. Lühmann for his strong efforts on the measurement software. Appreciation is given to the members of the Max-Planck Institutes 
mechanical workshop J. Faltin, R. Lenk, and J. Scharsach, who have manufactured all precision parts of the new dilatometer. This work is supported by the German Science Foundation through Project Nos. KU 3287/1-1 and GE1640/8-1.

${ }^{1}$ E. Grüneisen, Ann. Phys. (Leipzig) 39, 257 (1912).

${ }^{2}$ T. H. K. Barron and G. K. White, Heat Capacity and Thermal Expansion at Low Temperatures (Kluwer Academic/Plenum, Dordrecht, New York, 1999), and references therein.

${ }^{3}$ G. White and J. G. Collins, J. Low Temp. Phys. 7, 43-75 (1972).

${ }^{4}$ G. White and M. Minges, Int. J. Thermophys. 18, 1269-1327 (1997).

${ }^{5}$ J. Genossar and M. O. Steinitz, Rev. Sci. Instrum. 61, 2469 (1990).

${ }^{6}$ L. Zhu, M. Garst, A. Rosch, and Q. Si, Phys. Rev. Lett. 91, 066404 (2003).

${ }^{7}$ R. Küchler, N. Oeschler, P. Gegenwart, T. Cichorek, K. Neumaier, O. Tegus, C. Geibel, J. Mydosh, F. Steglich, L. Zhu, and Q. Si, Phys. Rev. Lett. 91, 066405 (2003).

${ }^{8}$ R. Küchler, P. Gegenwart, K. Heuser, E. W. Scheidt, G. R. Stewart, and F. Steglich, Phys. Rev. Lett. 93, 096402 (2004).

${ }^{9}$ R. Küchler, P. Gegenwart, J. Custers, O. Stockert, N. Caroca-Canales, C. Geibel, J. Sereni, and F. Steglich, Phys. Rev. Lett. 96, 256403 (2006).

${ }^{10}$ R. Küchler, T. Bauer, M. Brando, and F. Steglich, Rev. Sci. Instrum. 83, 095102 (2012).

${ }^{11}$ J. J. Neumeier, R. K. Bollinger, G. E. Timmins, C. R. Lane, R. D. Krogstad, and J. Macaluso, Rev. Sci. Instrum. 79, 033903 (2008).

${ }^{12}$ G. M. Schmiedeshoff, A. W. Lounsbury, D. J. Luna, S. J. Tracy, A. J. Schramm, S. W. Tozer, V. F. Correa, S. T. Hannahs, T. P. Murphy,
E. C. Palm, A. H. Lacerda, S. L. Bud'ko, P. C. Canfield, J. L. Smith, J. C. Lashley, and J. C. Cooley, Rev. Sci. Instrum. 77, 123907 (2006).

${ }^{13}$ M. Rotter, H. Müller, E. Gratz, M. Doerr, and M. Loewenhaupt, Rev. Sci. Instrum. 69, 2742 (1998).

${ }^{14}$ J. H. Park, D. Graf, T. P. Murphy, G. M. Schmiedeshoff, and S. W. Tozer, Rev. Sci. Instrum. 80, 116101 (2009).

${ }^{15}$ R. Pott and R. Schefzyk, J. Phys. E: Sci. Instrum. 16, 444 (1983).

${ }^{16}$ A. Steppke, R. Küchler, S. Lausberg, E. Lengyel, L. Steinke, R. Borth, T. Lühmann, C. Krellner, M. Nicklas, C. Geibel, F. Steglich, and M. Brando, Science 339, 933 (2013).

${ }^{17}$ R. Küchler, C. Stingl, and P. Gegenwart, Rev. Sci. Instrum. 87, 073903 (2016).

${ }^{18}$ S. Yomo, J. Phys. Soc. Jpn. 47(5), 1486 (1979).

${ }^{19}$ M. Doerr, W. Lorenz, T. Neupert, M. Loewenhaupt, N. V. Kozlova, J. Freudenberger, M. Bartkowiak, E. Kampert, and M. Rotter, Rev. Sci. Instrum. 79, 063902 (2008).

${ }^{20}$ R. Daou, F. Weickert, M. Nicklas, F. Steglich, A. Haase, and M. Doerr, Rev. Sci. Instrum. 81, 033909 (2010).

${ }^{21}$ V. C. Correa, S. Francoual, M. Jaime, N. Harrison, T. P. Murphy, E. C. Palm, S. W. Tozer, A. H. Lacerda, P. A. Sharma, and J. A. Mydosh, Phys. Rev. Lett. 109, 246405 (2012).

${ }^{22}$ M. Rotter, A. Barcza, M. Doerr, M. D. Le, J. Brooks, E. Jobiliong, and J. Perenboom, Phys. Rev. B 76, 144421 (2007).

${ }^{23}$ S. A. J. Wiegers, J. Rook, A. den Ouden, J. A. A. J. Perenboom, and J. C. Maan, IEEE Trans. Appl. Supercond. 22(3), 4301504 (2012).

${ }^{24}$ F. J. P. Wijnen, S. A. J. Wiegers, J. M. H. van Velsen, J. Rook, A. den Ouden, J. A. A. J. Perenboom, and N. E. Hussey, IEEE Trans. Appl. Supercond. 26(4), 4302505 (2016).

${ }^{25}$ J.-P. Michenaud, B. Lenoir, and C. Bellouard, Phys. Rev. B 26, 2552 (1982). 